\title{
THE USE OF INJECTABLE PLATELET RICH FIBRIN IN CONJUNCTION TO GUIDED BONE REGENERATION FOR THE MANAGEMENT OF WELL CONTAINED RIDGE DEFECT AT THE TIME OF EXTRACTION
}

\author{
Lydia N. Melek* and Maha R. Taalab**
}

\begin{abstract}
Background: Osseous defects resulting from periodontitis or from prior extracted teeth can complicate implant placement and/or restorative treatment. Grafting of these sites can develop better ridge morphology for implant placement or to support either fixed or removable prosthetics.

Objectives: This study aims to evaluate the effect of using injectable platelet-rich fibrin as adjunctive to Genesis bone graft and collagen membrane on the management of contained ridge defect at the time of extraction prior to implant placement. Moreover it compares this effect to that resulting from using the same bone graft and membrane alone.
\end{abstract}

Patients and methods: This study was conducted on fourteen patients selected from the outpatient clinic of the Faculty of Dentistry, Alexandria University. The Patients were divided equally into two groups: Group I (study group): consists of seven patients with severe maxillary anterior ridge defects. Ridge augmentation was performed using Genesis bone graft combined with injectable platelet-rich fibrin, followed by application of absorbable membranes to stabilize and protect the grafts. Group II (control group): consists of seven patients with severe maxillary anterior ridge defects. Ridge augmentation was performed using Genesis bone graft only, followed by application of absorbable membranes to stabilize and protect the grafts.

Results: there was a statistically significant difference between the 2 groups regarding the percent of change in alveolar bone width and density between preoperative and six months postoperative. However, the percent of change in the height of the labial plate of bone showed statistically insignificant difference between the 2 groups.

Conclusion: Guided bone regeneration before implant placement either at time of extraction or after extraction to avoid further bone resorption, helps to increase the alveolar ridge dimensions and improve its morphology (staged approach). The adjuvant use of injectable PRF with its high growth factor content may contribute to more favourable and predictable bone formation at the grafted site.

* Lecturer of Oral and Maxillofacial Surgery, Faculty of Dentistry, Alexandria University

** Lecturer of Periodontology and Oral medicine, Faculty of Dentistry, Alexandria University 


\section{INTRODUCTION}

Osseous defects resulting from periodontitis or from prior extracted teeth can complicate implant placement and/or restorative treatment. Grafting of these sites can develop better ridge morphology for implant placement or to support either fixed or removable prosthetics. ${ }^{(1)}$

Post-extraction crestal bone resorption is common and unavoidable which can lead to significant ridge dimensional changes. To regenerate enough bone for successful implant placement, Guided Bone Regeneration (GBR) is often required. GBR is a surgical procedure that uses barrier membranes with or without particulate bone grafts or/and bone substitutes. There are two approaches of GBR in implant therapy: GBR at implant placement (simultaneous approach) and GBR before implant placement either at time of extraction or after extraction to avoid further resorption, increase the alveolar ridge and improve its morphology (staged approach). Angiogenesis and ample blood supply play a critical role in promoting bone regeneration..$^{(2)}$

Bone regeneration can be accomplished through three different mechanisms: osteogenesis, osteoinduction, and osteoconduction. Osteogenesis is the formation and development of bone, even in the absence of local undifferentiated mesenchymal stem cells. Osteoinduction is the transformation of undifferentiated mesenchymal stem cells into osteoblasts or chondroblasts through growth factors that exist only in living bone. Osteoconduction is the process that provides a bio-inert scaffold, or physical matrix, suitable for the deposition of new bone from the surrounding bone or encourage differentiated mesenchymal cells to grow along the graft surface. ${ }^{(3)}$

Alloplasts are an inert synthetic graft material. The most commonly used alloplast materials are calcium carbonate, calcium sulfate, bioactive glass polymers and ceramic materials, including synthetic hydroxyapatite and tricalcium phosphate
(TCP). The mechanism of action of these materials is strictly osteoconduction. They provide a scaffold for enhanced bone tissue repair and growth. ${ }^{(3)}$

Studies by Dahlin et al. showed that if a barrier membrane was placed in direct contact with the surrounding bone surface and a space was created, only cells from the neighboring bone or bone marrow can migrate into this bone defect, without in-growth of competing soft tissue cells from the overlying mucosa. There may be additional benefits to the use of a membrane, such as protection of the wound from mechanical disruption and salivary contamination. A barrier membrane should satisfy the following conditions: tissue adhesion without mobility, block soft tissue in-growth, easy to use, maintains a space, and biocompatibility. Currently, barrier membranes are of two types, non-resorbable and resorbable. ${ }^{(4)}$

Most of the commercially available collagen membranes are developed from type I collagen or a combination of type I and type III collagen. The source of collagen comes from tendon, dermis, skin or pericardium of bovine, porcine or human origin. ${ }^{(5)}$ There are several advantages of collagen materials for use a barrier membrane to include: hemostasis $^{(6)}$, chemotaxis for periodontal ligament fibroblasts (7) and gingival fibroblasts (8), weak immunogenicity (9), easy manipulation and adaption, a direct effect on bone formation (10), and ability to augment tissue thickness ${ }^{(11)}$. Hence, collagen material appears to be an ideal choice for a bioresorbable GTR or GBR barrier.

The search for ways to accelerate new bone formation is a constant in the medical and dental areas. Thus, there has been research on the influence of blood cells on biomaterials applied in the human body. This evolution stems from the late 1990s in the last century, with the release of platelet-rich plasma (PRP) ${ }^{(12)}$, followed by the second generation of platelet aggregates, platelet-rich fibrin (PRF) ${ }^{(13)}$, until the recent advanced platelet-rich fibrin clot (a-PRF). ${ }^{(14)}$ 
These platelet concentrates propose an acceleration in healing of soft and hard tissues by increasing the concentration of growth factors such as transforming growth factor-b (TGF-b), insulinlike growth factor-1 (IGF-1), platelet-derived growth factor (PDGF), vascular endothelial growth factor (VEGF), fibroblast growth factor (FGF), epidermal growth factor (EGF) and platelet-derived epidermal growth factor (PDEGF). ${ }^{(15-17)}$

According to Choukron et al. PRF was initially used in implant surgery to enhance the healing properties of the bone. ${ }^{(17)} \mathrm{PRF}$ can promote the healing of osseous defects by the following mechanisms. According to Chang et al. PRF promotes the expression of phosphorylate extracellular signalregulated protein kinase (p-ERK) and stimulates the production of osteoprotegerin (OPG) which in turn causes proliferation of osteoblasts. ${ }^{(18)}$

Another study by Huang et al. reported that PRF stimulates the osteogenic differentiation of the human dental pulp cells by upregulating osteoprotegerin and alkaline phosphatase expression. ${ }^{(19)}$

PRF also releases growth factors such as plateletderived growth factor and transforming growth factor which promote periodontal regeneration. ${ }^{(20,21)}$ Chang et al. in a study reported that PRF stimulates cell proliferation in a specific manner. PRF induces cell proliferation of osteoblasts, periodontal ligament cells and growth factors during a 3-day culture period and suppressed oral epithelial cell growth. These cell type-specific actions may be beneficial for periodontal regeneration. ${ }^{(22)}$

Diss et al. in a 1 year prospective study on osteotome sinus floor elevation using Choukroun's platelet-rich fibrin grafting material clearly demonstrated that fibrin matrix of PRF directly promotes angiogenesis. ${ }^{(23)}$

Moreover it was proved that PRF has an inherent osteoconductive and/or osteoinductive property which is beneficial for regeneration of the bone as Sanchez et al. in an experimental study compared the influence of PRP and PRF on proliferation and differentiation of osteoblasts and he reported that the affinity of osteoblasts to the PRF membrane appeared to be superior than the affinity of osteoblasts to PRP. ${ }^{(24)}$

The use of platelet aggregates in injectable form is widespread, especially in orthopedics ${ }^{(25)}$ and in plastic surgery ${ }^{(26)}$, where it was possible to obtain favorable results, but these concentrates use venous blood collection tubes with anticoagulants or separating gel. However, the tubes used in this technique have no additives that interfere in the process.

This study aims to evaluate the effect of using injectable platelet-rich fibrin as adjunctive to Genesis bone graft and collagen membrane on the management of contained ridge defect at the time of extraction prior to implant placement. Moreover it compares this effect to that resulting from using the same bone graft and membrane alone.

\section{PATIENTS AND METHODS}

\section{PATIENTS}

\section{Study design:}

This study was conducted on fourteen patients selected from the outpatient clinic of the Faculty of Dentistry, Alexandria University. Healthy partially edentulous patients who were seeking prosthetic rehabilitation of missing maxillary anterior teeth and suffering from locally resorbed maxillary ridge, presenting a problem for dental implant insertion, were candidates for the present study. All patients were informed about the aim of the designed study and an informed consent was obtained.

The Patients were divided equally into two groups:

\section{Group I (study group)}

This group consists of seven patients with severe maxillary anterior ridge defects, requiring horizontal bone reconstruction in the anterior maxilla. Ridge 
augmentation was performed using Genesis bone graft combined with injectable platelet -rich fibrin, followed by application of absorbable membranes to stabilize and protect the grafts.

\section{Group II (control group)}

This group consists of seven patients with severe maxillary anterior ridge defects, requiring horizontal bone reconstruction in the anterior maxilla. Ridge augmentation was performed using Genesis bone graft only, followed by application of absorbable membranes to stabilize and protect the grafts.

\section{Inclusion criteria}

- Patients' age ranging from 20-50 years old.

- Patients with severe maxillary anterior ridge defects

\section{Exclusion criteria}

- Medically compromised patients with systemic diseases contraindicating surgery.

- Dental history of bruxism and parafunctional habits.

- Untreated periodontitis.

- Bone diseases that affect bone formation.

- Patients with insufficient interocclusal space for implant placement.

- Smokers.

\section{Informed consent}

An informed consent was obtained from the patients. All the procedures were described in detail including the benefits and side effects. It was also mentioned that the patient has the right of withdrawal from the study anytime according to their wish.

\section{MATERIALS}

1. Resorbable collagen membrane $(15 \times 20 \times 0.3$ mm) T-Gen [Bioland , Korea]
2. Genesis: (500-1000 $\mu \mathrm{m}-1 \mathrm{~g})$ beta tri calcium phosphate + hydroxyapatite, bone alloplast grafting material [Dio Implant, Korea].

3. Injectable platelet-rich fibrin.

\section{i- PRF preparation:}

i-PRF is the next step PRF and offers the characteristics of A-PRF as a liquid. On top i-PRF capture an amount of stem cells from the blood stream. The use of i-PRF is in its infancy, but already, the results in both oral surgery and in regenerative medicine are very promising.

Injectable Platelet rich fibrin (PRF) was prepared according to the protocol developed by Choukroun. (27) Venous blood was drawn into i-PRF ${ }^{\mathrm{TM}} 10 \mathrm{ml}$ tubes. This was done fast and each tube was placed into the centrifuge. The centrifuge was adjusted to $700 \mathrm{rpm}$ and the tubes were spinned for 3 minutes. The spin has separated the RBC's and the i-PRF was visible at the top part of the tube. Upon termination of this process, it was possible to observe an orange color area in the upper part of the tube (i-PRF) and the remaining blood materials below. Then, the tube was opened carefully, to avoid homogenization of the material. We collected about $3 \mathrm{ml}$ of i-PRF from the tubes using a $5 \mathrm{ml}$ syringe. The liquid (i-PRF) was drawn into the syringe by careful suction. When the i-PRF was injected onto the bone graft, it clotted after few seconds and encapsulated the particles in a very nice way, allowing to carve the bone graft and give it a compact form as if it is "sticky bone". (Figure 1)

\section{METHODS}

\section{Presurgical phase:}

All patients went for preoperative history and clinical examination: Patients' data were collected; name, gender and age, the medical and dental history were accurately taken. The oral mucosa of the edentulous area was examined for color, texture, firmness and thickness. 
Also, preoperative evaluation for all patients included cone beam computerized tomography (CBCT), to evaluate the residual ridge height (labially and palatally) and width (at a point $3 \mathrm{~mm}$ from the midpoint of the alveolar crest), and to evaluate the density of bone (at the same fixed point; $3 \mathrm{~mm}$ from the midpoint of the alveolar crest) .

\section{Surgical phase}

All patients were operated under local anesthesia, secured with Mepivacaine 2\% (Mepecaine HCL 2\% with Levonordin 1: 20,000. Alexandria Company for Pharmaceutics and Chemical Industties, Alexandria), and under strict aseptic conditions.

A pyramidal full thickness mucoperiosteal flap was designed in the edentulous area using Bard Parker blade number 15. The flap was then reflected to expose the alveolar bone. Removal of any remaining roots was then performed followed by curettage and proper debridement of the socket to remove any inflamed tissues. (Figure 2)

In the control group, the graft was directly packed to fill the bone defect then covered by the collagen membrane to stabilize it in place. (Figure 3)

While in the study group, the graft was first mixed with the injectable PRF, allowing it to become compact and moldable (sticky bone). It was then applied to the bone defect and covered with the collagen membrane. (Figure 4)

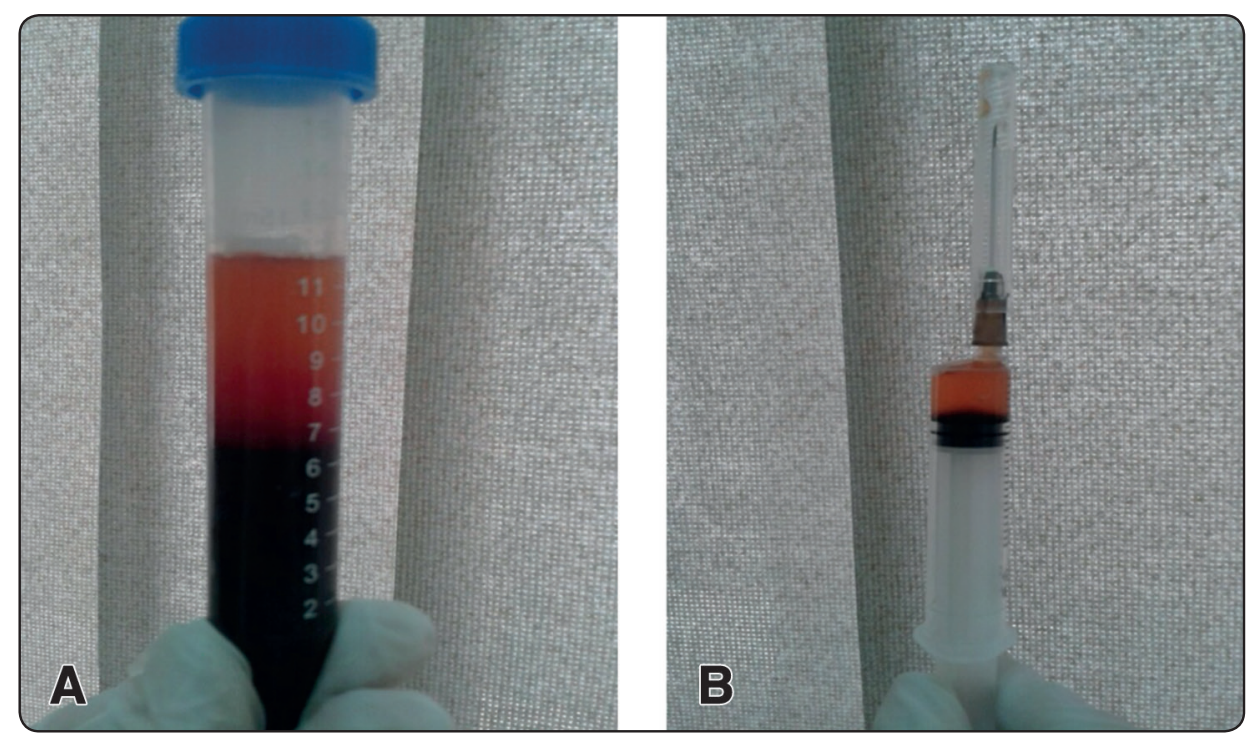

Fig. (1) (A) Injectable PRF preparation, i-PRF after being drawn from the test tube with a syringe and ready for use.

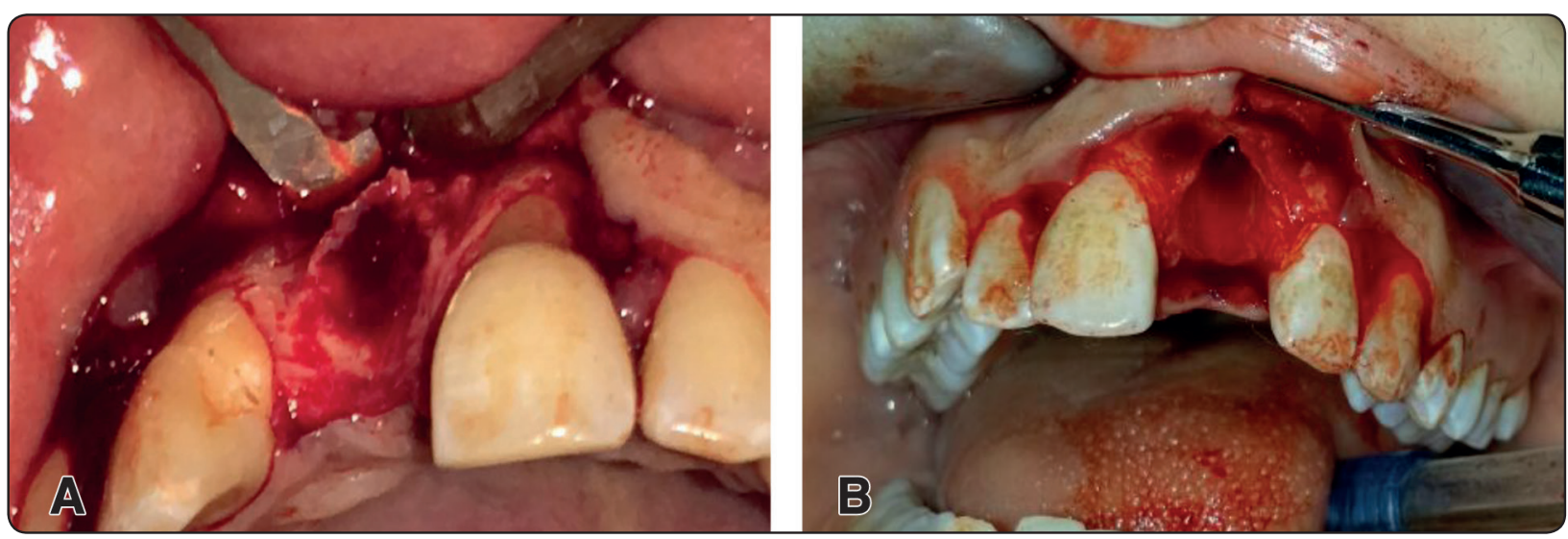

Fig. (2) Osseous defect after root extraction in (A) study case, and (B) control case 


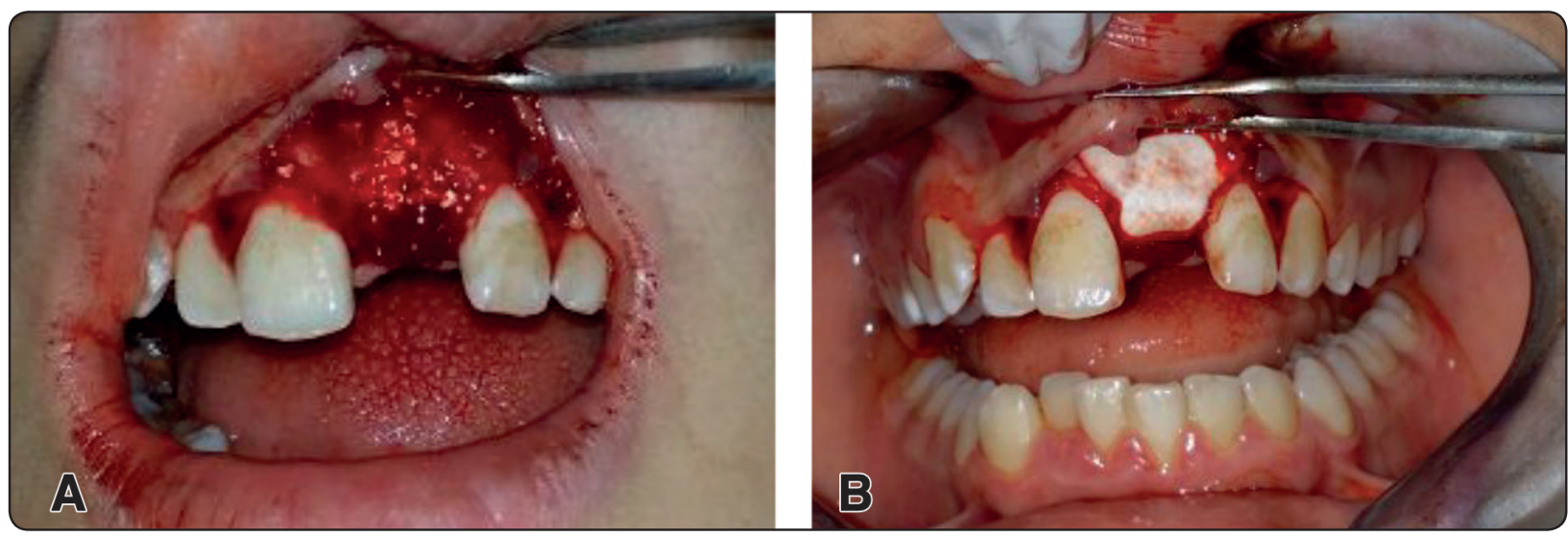

Fig. (3) (A) Graft filling the bone defect in the control group, (B) graft covered by collagen membrane in the control group.

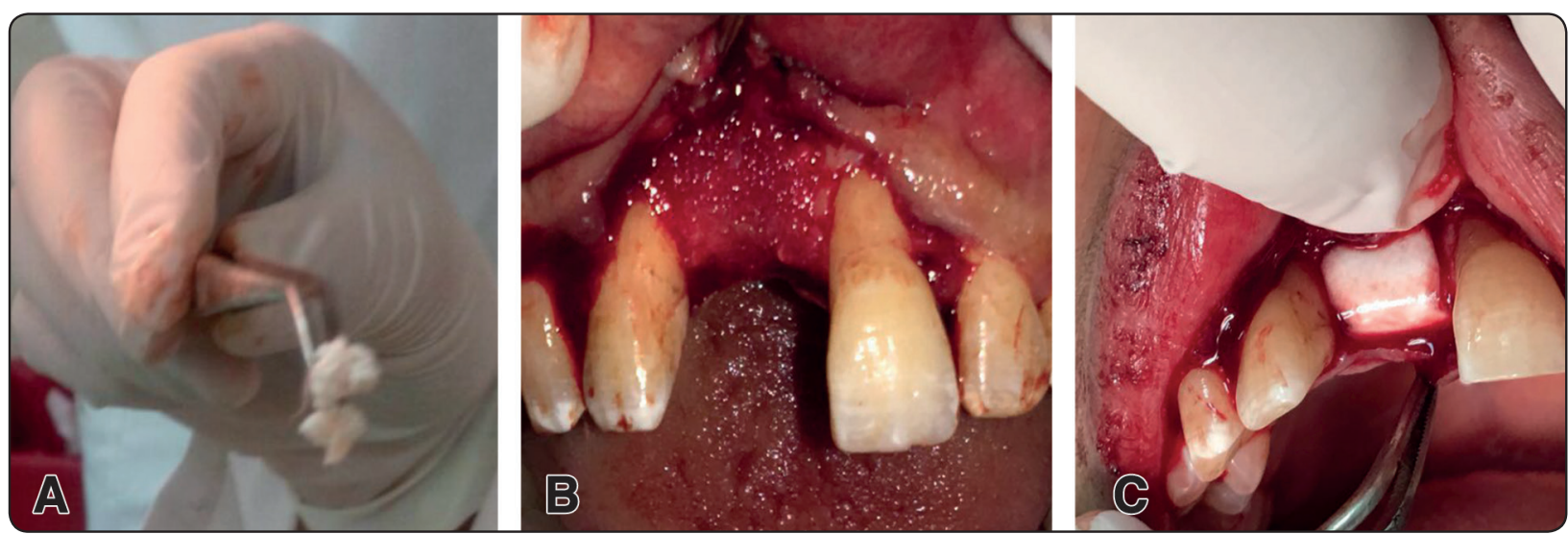

Fig. (4) Study group: (A) graft after being mixed with i-PRF, (B) graft applied to the defect, and (C) covered with a resorbable membrane.

The flap was then repositioned and sutured using 3-0 black silk suture material in patients of both groups.

\section{Postsurgical phase:}

Postoperative instructions were given to the patients including cold packs on the first day, then warm mouth wash for the following days beside oral hygiene instructions. An antibiotic; Amoxicillin \& Clavulanate Potassium 1gm (Augmentin 1g , GlaxoSmithKline. Cairo, Egypt) was prescribed every 12 hours for 5 days. Non-steroidal antiinflammatory drug; Diclofenac Potassium 50mg (Cataflam 50 mg tablets: manufactured by Novartis Pharma Company, Egypt) was prescribed 3 times daily for 3 days postoperatively. Chlorohexidine mouthwash (Hexitol MW, Arab drug company, Cairo, Egypt) was also prescribed for 7 days after the surgery.

\section{Postoperative evaluation}

\section{Clinical evaluation:}

All patients were examined 7 days after surgery at which time the sutures were removed then weekly for the first month postoperatively, then on intervals of 1, 3 and 6 months postoperatively. The clinical parameters of importance included: pain, tenderness, discomfort, wound dehiscence, graft particles leakage or any other complications at the site of surgery. 


\section{Radiographic evaluation:}

CBCT was obtained at 3 and 6 months postoperatively for all patients to measure bone density, and the alveolar ridge dimensions (ridge width and height) at the same reference points used preoperatively thus indicating the amount of the newly formed bone.

\section{Statistical analysis of the data}

Data were fed to the computer and analyzed using IBM SPSS software package version 20.0. Student t-test was used to compare two groups for normally distributed quantitative variables. Paired t-test and ANOVA with repeated measures and Post Hoc test (LSD) was assessed for comparison between different periods. Pearson coefficient was used to correlate between quantitative variables. Significance of the obtained results was judged at the $5 \%$ level.

\section{RESULTS}

This study was conducted on fourteen partially edentulous patients selected from the outpatient clinic of the Faculty of Dentistry, Alexandria University, who were seeking prosthetic rehabilitation of missing maxillary anterior teeth and suffering from locally resorbed maxillary ridge, presenting a problem for dental implant insertion.

The selected fourteen participants were divided equally into two groups; control group and study group each consisted of seven patients. The study group included 6 females and 1 male, while the control group included 5 females and 2 males, the age range for both groups was from 25 to 55 years old.

\section{Clinical results:}

Normal color of the oral mucosa, proper healing and adequate closure of the wound were achieved in patients of both groups. No signs of infection, graft particle leakage or other complications were observed in any patient within the two groups throughout the postoperative follow up period.

\section{Radiographic results:}

\section{Width of the alveolar ridge: [Figures 5,6]}

Regarding the alveolar bone width (Table 1), a statistical significant increase of alveolar bone width in both groups at three months postoperative compared with the bone width preoperatively was observed. Also at 6 months postoperative, there was a significant increase of alveolar bone width in both groups compared to that at 3 months postoperative. The percentage of change in alveolar bone width between preoperative and 6 months postoperative in the control group was $1.4 \pm 0.4 \%$ while in the study group, it was higher; $2.9 \pm 0.6 \%$. There was a statistically significant difference between the two groups $(\mathrm{p}=<0.001)$.

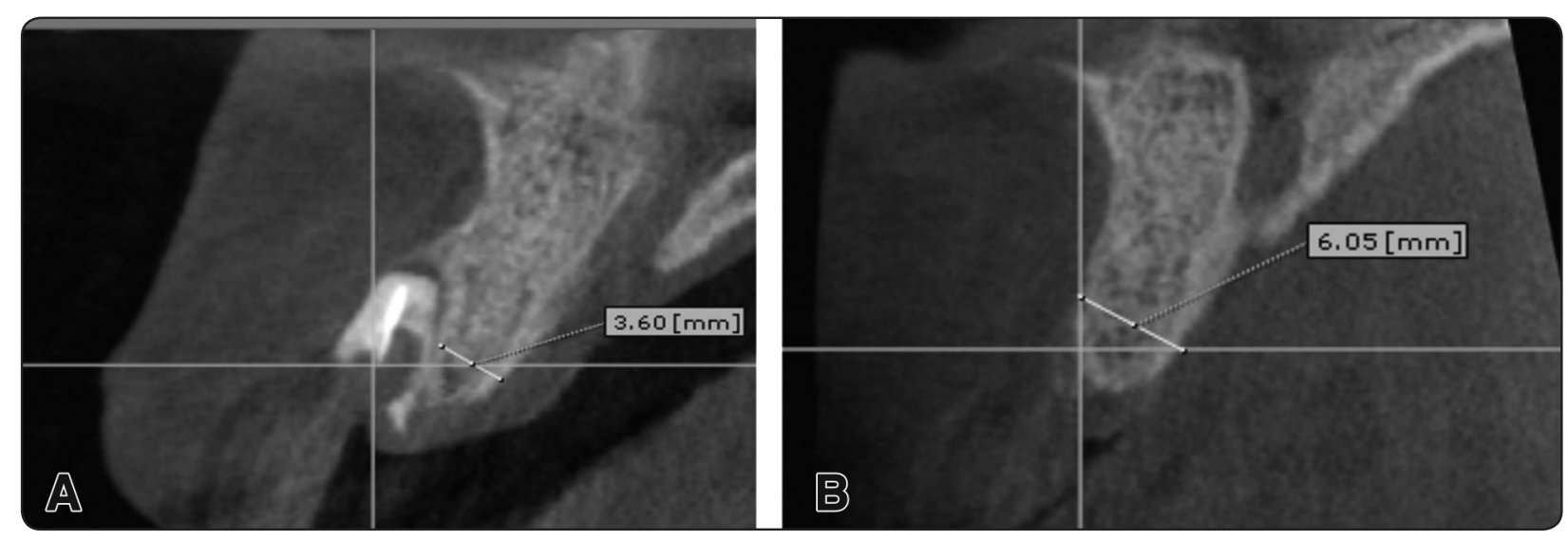

Fig. (5) Width of the alveolar ridge preoperatively (A) and at 6 months postoperatively (B) in a case of the study group. 


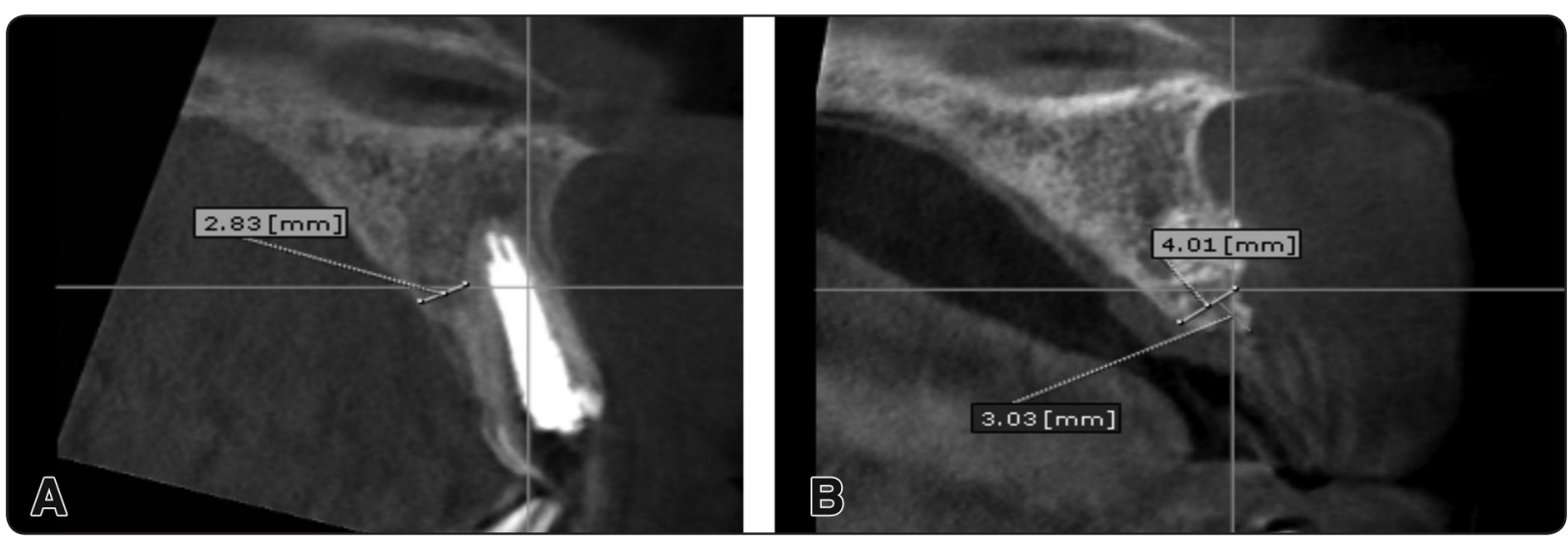

Fig. (6) Width of the alveolar ridge preoperatively (A) and at 6 months postoperatively (B) in a case of the control group.

\section{Density of bone}

Regarding the density of bone (Table 1), a statistical significant increase of alveolar bone density in both groups at three months postoperative compared with the bone density preoperatively was observed, with no significant difference between the two groups. At 6 months postoperative, there was a significant increase of alveolar bone density in both groups compared to that at 3 months postoperative. The percentage of change in alveolar bone density between preoperative and 6 months postoperative in the control group was $129.7 \pm 0.8 \%$ while in the study group, it was higher; $347.7 \pm 9.6 \%$. There was a statistically significant difference between the two groups $(\mathrm{p}=<0.001)$.

\section{Height of the labial plate of bone:}

Regarding the alveolar bone height labially (Table 1), a statistical significant increase of alveolar bone height in both groups at three months postoperative compared with the bone height preoperatively was observed. Also at 6 months postoperative, there was a significant increase of alveolar bone height in both groups compared to that at 3 months postoperative. The percentage of change in alveolar bone height between preoperative and 6 months postoperative in the control group was $4.6 \pm 0.4 \%$ while in the study group, it was $4.3 \pm 0.4 \%$. There was no statistically significant difference between the two groups ( $\mathrm{p}=0.145)$.
TABLE (1) Comparison between the studied groups according to different parameters

\begin{tabular}{|c|c|c|c|}
\hline $\begin{array}{c}\text { Bone } \\
\text { measurements }\end{array}$ & Control & Study & $\mathbf{p}$ \\
\hline $\begin{array}{l}\text { Width of } \\
\text { alveolar ridge }\end{array}$ & $(n=7)$ & $(n=7)$ & \\
\hline Pre & $3.1 \pm 0.5$ & $3.1 \pm 0.6$ & 0.894 \\
\hline After $3 \mathrm{~m}$ & $6.7^{\mathrm{a}} \pm 0.6$ & $5.2^{\mathrm{a}} \pm 0.8$ & $0.001^{*}$ \\
\hline After $6 \mathrm{~m}$ & $4.5^{\mathrm{ab}} \pm 0.6$ & $6.0^{\mathrm{ab}} \pm 0.5$ & $<0.001^{*}$ \\
\hline $\begin{array}{l}\text { D(Pre - After } \\
6 \mathrm{~m})\end{array}$ & $1.4 \pm 0.4$ & $2.9 \pm 0.6$ & $<0.001^{*}$ \\
\hline Density of bone & $(\mathbf{n}=7)$ & $(n=7)$ & \\
\hline Pre & $1223.4 \pm 3.9$ & $1247.5 \pm 157.4$ & 0.700 \\
\hline After $3 \mathrm{~m}$ & $1300.6^{\mathrm{a}} \pm 4.2$ & $1264.8^{\mathrm{a}} \pm 161.0$ & 0.578 \\
\hline After $6 \mathrm{~m}$ & $1353.1^{\mathrm{ab}} \pm 4.0$ & $1595.2^{\mathrm{ab}} \pm 167.1$ & $0.009^{*}$ \\
\hline $\begin{array}{l}\text { D(Pre - After } \\
6 \mathrm{~m})\end{array}$ & $129.7 \pm 0.8$ & $347.7 \pm 9.6$ & $<0.001^{*}$ \\
\hline $\begin{array}{l}\text { Height of labial } \\
\text { plate of bone }\end{array}$ & $(n=7)$ & $(n=7)$ & \\
\hline Pre & $10.0 \pm 0.5$ & $13.8 \pm 0.8$ & $<0.001^{*}$ \\
\hline After $3 \mathrm{~m}$ & $13.7^{\mathrm{a}} \pm 0.5$ & $15.9^{\mathrm{a}} \pm 1.3$ & $0.001^{*}$ \\
\hline After $6 \mathrm{~m}$ & $14.6^{\mathrm{ab}} \pm 0.5$ & $18.1^{\mathrm{ab}} \pm 0.8$ & $<0.001^{*}$ \\
\hline $\begin{array}{l}D(\text { Pre - After } \\
6 \mathrm{~m})\end{array}$ & $4.6 \pm 0.4$ & $4.3 \pm 0.4$ & 0.145 \\
\hline
\end{tabular}

Data was expressed in mean $\pm S D$

a: Statistically significant with pre

b: Statistically significant with $3 \mathrm{~m}$

p: p value for comparing between test and control

*: Statistically significant at $p \leq 0.05$ 


\section{DISCUSSION}

The i-PRF is a new alternative to the platelet aggregate to different areas of Medicine and Dentistry, enabling experts to further research this product. Because it is autogenous, it decreases the chances of adverse reactions to the implanted material, especially immune-mediated ones, as with other types of grafting ${ }^{(28)}$, which qualifies it as a viable option in regenerative procedures.

The possibility of bonding of i-PRF with biomaterials for bone grafting creates an alternative to PRP as a platelet aggregate for bone regeneration. PRP is used in regenerative procedures because of the possibility to optimize bone formation ${ }^{(29)}$. The technique presented in this study permits incorporation of the graft without the use of anticoagulants or other additives, thereby forming a well-agglutinated "sticky bone for bone grafting", as shown.

The literature includes few studies using iPRF and graft materials. Choukroun J (2014) ${ }^{(27)}$ was the pioneer in performing and using iPRF in adjunction to bone grafts in bone augmentation around dental implants .

This study was conducted on fourteen partially edentulous patients selected from the outpatient clinic of the Faculty of Dentistry, Alexandria University, who were seeking prosthetic rehabilitation of missing maxillary anterior teeth and suffering from locally desorbed maxillary ridge, presenting a problem for dental implant insertion.

The selected fourteen participants were divided equally into two groups; control group and study group each consisted of seven patients. In the study group, ridge augmentation was performed using Genesis bone graft combined with injectable platelet -rich fibrin, followed by application of absorbable membranes to stabilize and protect the grafts. In the control group, ridge augmentation was performed using Genesis bone graft only, followed by application of absorbable membranes to stabilize and protect the grafts.
Clinically, the patients of both groups showed uneventful healing without complications throughout the postoperative follow-up period.

Radiographically, the alveolar bone density and the alveolar ridge dimensions (ridge width and height) were assessed at 3 and 6 months postoperatively. Measurements were done at the same reference points used preoperatively (marked at a height of $3 \mathrm{~mm}$ from the midpoint of the alveolar crest) thus indicating the amount of the newly formed bone.

Regarding the change in bone density from preoperative to the third postoperative month and from the third postoperative to the sixth postoperative month, there was an increase in bone density in both study and control groups, but this increase in bone density was greater in the study group than in the control group. The difference between the two groups was statistically significant at the sixth postoperative month and in the percent of change [D(Pre - After 6m)]. Regarding the alveolar bone width, a statistical significant increase of alveolar bone width in both groups at three months postoperative compared with the bone width preoperatively was observed. Also at 6 months postoperative, there was a significant increase of alveolar bone width in both groups compared to that at 3 months postoperative. There was a statistically significant difference between the two groups regarding the percentage of change in alveolar bone width between preoperative and 6 months postoperative in favor of the study group.

This is in agreement with the findings of Singh et al. $(2012)^{(30)}$, who found in their study that PRF has significantly improved soft tissue healing, bone regeneration and increase in bone density in extraction sockets.

Also, Girish et al. (2013) ${ }^{(31)}$ found in their study a definite improvement in the regeneration of bone after third molar surgery in cases treated with PRF as compared to the control group post-operatively. They also stated that this increase in the bone 
density signifies and highlights the use of PRF, certainly as a valid method in accelerating hard tissue regeneration.

Moreover, Choukron has demonstrated that PRF can be used in conjunction with bone grafts offering several advantages such as promotion of wound healing, bone growth and maturation, graft stabilization, wound sealing and hemostasis and improving the handling properties of graft materials. ${ }^{(32)}$

Our results were close to those concluded by Tatullo et al. ${ }^{(33)}$ who conducted histological and clinical evaluations of 60 patients who underwent sinus lifting surgery before implant surgery. The experimental group received bovine bone graft material (Bio-Oss) combined with PRF, whereas the control group received only bovine bone graft material(Bio-Oss, Geistlich Pharma AG, Wolhusen, Switzerland).The study made histologic and histomorphometric evaluations on days 106,120, and150.The results revealed that the good osteoconductive capacity of PRF led to the production of new bone, even at106 days. No implant loss was observed at 36 months. Primary implant stability, assessed by means of resonance frequency analysis, did not show any statistical difference between test and control groups.

Moreover, Ozdemir et al ${ }^{(34)}$ assessed the effects of PRF on bone augmentation in an animal model. Surgically created defects were filled with PRF, $\mathrm{BCP}$, or anorganic bovine bone (ABB) and were covered with titanium membranes. Control groups were left empty. Histomorphometric evaluation was carried out at 1 and 3 months. The control group showed the least new bone formation, and similar new bone are as were found among PRF, BCP, and ABB groups after 1 month. PRF and $\mathrm{ABB}$ showed a greater area of new bone formation than the other 2 groups at 3 months. This proved that PRF by itself can induce bone formation.

Contrary to our results and the previous studies results which concluded that PRF can induce more new bone formation than bone grafts alone, Yeniyol S. et al (2013) ${ }^{(35)}$ performed a histological study to evaluate the efficacy of PRF mixed with biphasic calcium phosphate(BCP) on bone regeneration in surgically created bone defects in the tibias of 6 sheep. The defects were left empty or grafted with BCP, PRF, or BCP and PRF. They observed that there was statistically higher new bone formation in the group with defects filled with PRF and BCP than the other groups on days 10 and 20. Also, at 40 days, there was relatively similar new bone in all 4 defects. They concluded that PRF is effective in the early stages of healing only.

However the results of our research proved that iPRF in addition to Gensis bone graft had favored the formation of more new bone with higher density than the use of the bone graft alone. The effectiveness of PRF in conjunction to bone graft depends not only on its features but also the properties of the coadministered grafting material. Moreover, it was reported that PRF dissolves more slowly than other platelet concentrates and that's why it can give chance for defect to get benefit of the growth factors in it for longer time than other platelet concentrates and so reaching excellent results in both bone quantity and quality. ${ }^{(33)}$

\section{CONCLUSION}

Guided bone regeneration before implant placement either at time of extraction or after extraction to avoid further bone resorption, helps to increase the alveolar ridge dimensions and improve its morphology (staged approach). The adjuvant use of injectable PRF with its high growth factor content may contribute to more favourable and predictable bone formation at the grafted site. Also, the addition of the injectable PRF to the bone graft made it in the form of "sticky bone" which facilities its application and stability in the ridge defect. 


\section{REFERENCES}

1. Kurtzman GM, Dompkowski DF, Mahesh L. Treatment of a Large Osseous Defect at Time of Extraction and Implant Placement .Inside Dentistry, $2015 ; 11: 1$.

2. Liu J, Kerns DG. Mechanisms of Guided Bone Regeneration: A Review. Open Dent J. 2014; 8: 56-65.

3. Misch CE, Dietsh F. Bone-grafting materials in implant dentistry. Implant Dent. 1993;2:158-67.

4. Dahlin C, Buser D, Dahlin C, Schenk R, editors. In: Guided bone regeneration in implant dentistry. Chicago.: IL: Quintessence Publ; 1994. Scientific Background of guided bone regeneration.

5. Bunyaratavej P, Wang HL. Collagen membranes: a review. J Periodontol. 2001;72:215-29.

6. Wang HL, Carroll MJ. Guided bone regeneration using bone grafts and collagen membranes. Quint Int. 2001; 32:504-15.

7. Postlethwaite AE, Seyer JM, Kang AH. Chemotactic attraction of human fibroblasts to type I II and III collagens and collagen-derived peptides. Proceed Nat Acad Sci USA. 1978;75:871-5.

8. Locci P, Calvitti M, Belcastro Setal. Phenotype expression of gingival fibroblasts cultured on membranes used in guided tissue regeneration. J Periodontol. 1997;68:857-63.

9. Schlegel AK, Mohler H, Busch Fetal. Preclinical and clinical studies of a collagen membrane (Bio-Gide). Biomaterials. 1997; 18:535-8.

10. Rothamel D, Schwarz F, Sculean Aetal. Biocompatibility of various collagen membranes in cultures of human PDL fibroblasts and human osteoblast-like cells. Clin Oral Implants Res. 2004;15:443-9.

11. Pitaru S, Tal H, Soldinger Metal. Collagen membranes prevent apical migration of epithelium and support new connective tissue attachment during periodontal wound healing in dogs. J Periodont Res. 1989; 24:247-53.

12. Marx RE, Carlson ER, Eichstaedt RM, Schimmele SR, Strauss JE, Georgeff KR. Platelet-rich plasma: growth factor enhancement for bone grafts. Oral Surg Oral Med Oral Pathol Oral Radiol Endod. 1998; 85(6):638-46.

13. Choukroun J, Adda F, Schoeffler C, Vervelle A. Une opportunité en paro-implantologie: Le PRF. Implantodontie. 2001;42:55-62.
14. Ghanaati S, Booms P, Orlowska A, Kubesch A, Lorenz J, Rutkowski J, et al. Advanced platelet-rich fibrin: a new concept for cell-based tissue engineering by means of inflammatory cells. J Oral Implantol. 2014;40(6):679-89.

15. Soffer E, Ouhayoun JP, Anagnostou F. Fibrin sealants and platelet preparations in bone and periodontal healing. Oral Surg Oral Med Oral Pathol Oral Radiol Endod. 2003;95(5):521-8.

16. He L, Lin Y, Hu X, Zhang Y, Wu H. A comparative study of platelet-rich fibrin (PRF) and platelet-rich plasma (PRP) on the effect of proliferation and differentiation of rat osteoblasts in vitro. Oral Surg Oral Med Oral Pathol Oral Radiol Endod. 2009;108(5):707-13.

17. Choukroun J, Adda F, Schoeffer C, Vervelle A.PRF: an opportunity in perio-implantology. Implantodontie. 2000; 42:55-62.

18. Chang IC, Tsai $\mathrm{CH}$, Chang YC. Platelet-rich fibrin modulates the expression of extracellular signal-regulated protein kinase and osteoprotegerin in human osteoblasts. $\mathrm{J}$ Biomed Mater Res A .2010; 95:327-32.

19. Huang FM, Yang SF, Zhao JH, Chang YC. Platelet-rich fibrin increases proliferation and differentiation of human dental pulp cells. J Endod 2010;36:1628e32.

20. Dohan DM, Choukroun J, Diss A, et al. Platelet-rich fibrin (PRF): a second-generation platelet concentrate. Part II: platelet related biologic features. Oral Surg Oral Med Oral Pathol Oral Radiol Endod 2006;101:E45e50.

21. Dohan DM, Choukroun J, Diss A, et al. Platelet-rich fibrin(PRF): a second-generation platelet concentrate. Part III: leucocyte activation: a new feature for platelet concentrates? Oral Surg Oral Med Oral Pathol Oral Radiol Endod 2006;101:E51e55.

22. Tsai CH, Shen SY, Zhao JH, Chang YC. Platelet-rich fibrin modulates cell proliferation of human periodontally related cellsin vitro. J Dent Sci 2009;4:130e5.

23. Diss A, Dohan DM, Mouhyi J, Mahler P. Osteotome sinus floor elevation using Choukroun's platelet-rich fibrin as grafting material: a 1-year prospective pilot study with microthreaded implants. Oral Surg Oral Med Oral Pathol Oral Radiol Endod 2008;105, 572e9.

24. Sanchez AR, Sheridan PJ, Kupp LI. Is platelet-rich plasma the perfect enhancement factor? A current review. Int J Oral Maxillofac Implants 2003;18:93-103.

25. Kon E, Filardo G, Di Martino A, Marcacci M. Platelet-rich plasma (PRP) to treat sports injuries: evidence to support 
its use. Knee Surg Sports Traumatol Arthrosc. 2011; 19(4):516-27.

26. Sclafani AP, Saman M. Platelet-rich fibrin matrix for facial plastic surgery. Facial Plast Surg Clin North Am. 2012; 20(2):177-86.

27. Choukroun J. Advanced PRF \& i-PRF: Platelet concentrates or blood concentrates? J Periodontal Med Clin Pract 2014; 01: 3.

28. Alijotas-Reig J, Fernández-Figueras MT, Puig L. Inflammatory, immune-mediated adverse reactions related to soft tissue dermal fillers. Semin Arthritis Rheum. 2013;43(2):241-58.

29. Chen TL, Lu HJ, Liu GQ, Tang DH, Zhang XH, Pan ZL, et al. Effect of autologous platelet-rich plasma in combination with bovine porous bone mineral and bio-guide membrane on bone regeneration in mandible bicortical bony defects. J Craniofac Surg. 2014;25(1):215-23.

30. Singh A, Kohil M, Gupta N. Platelet rich fibrin: a noval approach for osseous regeneration. J Maxillofac Oral Surg 2012; 11(4): 430-4
31. Girish Rao S, Preethi Bhat, Nagesh KS, Gundu H, et al. Bone Regeneration in Extraction Sockets with Autologous Platelet Rich Fibrin Gel. J Maxillofac Oral Surg 2013; 12(1): 11-6.

32. Choukron J, Diss A, Simponieri A, Girard MO, Schoeffler $\mathrm{C}$, Dohan $\mathrm{S}$ et al. Platelet-rich fibrin (PRF): a secondgeneration platelet concentrate. Part IV: clinical effects on tissue healing. Oral Surg Oral Med Oral Pathol Oral Raiol Endod 2006; 101: 56-60.

33. Tatullo M, Marrelli M, Cassetta M, etal. Platelet rich fibrin $(\mathrm{PRF})$ in reconstructive surgery of atrophied maxillary bones: clinical and histological evaluations. Int JMedSci. 2012; 9:872-880.

34. Ozdemir H, Ezirganli S, IsaKara M, et al. Effectsofplateletrich fibrin alone used with rigid titanium barrier. Arch Oral Biol. 2013; 58:537-544.

35. Yeniyol S., Tekkesin M., Atunatmaz K. The Use of Platelet-Rich Fibrin in Combination With Biphasic Calcium Phosphate in the Treatment of Bone Defects :A Histologic and Histomorphometric Study.Current TherapeuticResearch. 2013;75:15-21. 